\title{
Maximizing Influence of Leaders in Social Networks
}

\author{
Xiaotian Zhou and Zhongzhi Zhang* \\ Shanghai Key Laboratory of Intelligent Information Processing, Fudan University, Shanghai 200433, China \\ School of Computer Science, Fudan University, Shanghai 200433, China \\ \{20210240043,zhangzz\}@fudan.edu.cn
}

\begin{abstract}
The operation of adding edges has been frequently used to the study of opinion dynamics in social networks for various purposes. In this paper, we consider the edge addition problem for the DeGroot model of opinion dynamics in a social network with $n$ nodes and $m$ edges, in the presence of a small number $s \ll n$ of competing leaders with binary opposing opinions 0 or 1 . Concretely, we pose and investigate the problem of maximizing the equilibrium overall opinion by creating $k$ new edges in a candidate edge set, where each edge is incident to a 1-valued leader and a follower node. We show that the objective function is monotone and submodular. We then propose a simple greedy algorithm with an approximation factor $\left(1-\frac{1}{e}\right)$ that approximately solves the problem in $O\left(n^{3}\right)$ time. Moreover, we provide a fast algorithm with a $\left(1-\frac{1}{e}-\epsilon\right)$ approximation ratio and $\tilde{O}\left(m k \epsilon^{-2}\right)$ time complexity for any $\epsilon>0$, where $\tilde{O}(\cdot)$ notation suppresses the poly $(\log n)$ factors. Extensive experiments demonstrate that our second approximate algorithm is efficient and effective, which scales to large networks with more than a million nodes.
\end{abstract}

\section{CCS CONCEPTS}

- Theory of computation $\rightarrow$ Graph algorithms analysis; Social networks; Discrete optimization; • Information systems $\rightarrow$ Data mining.

\section{KEYWORDS}

Opinion dynamics, social network, multi-agent system, graph algorithm, influence maximization, discrete optimization, Laplacian solver

\section{INTRODUCTION}

It has been heavily studied in the community of social sciences how opinions evolve and shape through social interactions among individuals with potentially different opinions $[15,18]$. In the current digital age, social media and online social networks provide unprecedented access to social interactions and individuals' opinions, leading to a fundamental change of the way people propagate, share, and shape opinions [25]. As such, in the past years the problem of opinion dynamics has received considerable attention from scientists in various disciplines [5, 16,21], including the AI community $[6,14,17]$. In order to understand the evolution and shaping of opinions, numerous models have been introduced [36], among which the DeGroot model [15] is a popular one. In the DeGroot model, the final opinions of individuals reach consensus. Since its

*Corresponding author. Zhongzhi Zhang is also with Shanghai Blockchain Engineering Research Center, as well as Research Institute of Intelligent Complex Systems, Fudan University, Shanghai 200433. establishment, the DeGroot model has been extended or generalized by incorporating different factors affecting opinion formation or agreement $[16,21]$.

Within the area of opinion dynamics, in addition to the development of models explaining the spread and formation of opinions, the problem of effectively shifting opinions in a social network has also become an active direction of study in recent years [1, 20, 29, $39,42]$, since it is closely related to various practical application settings, such as public health campaigns, political candidates, and product marketing. Most of previous work focus on the operations on individuals in a social network so as to optimize different opinions, for example, to maximize or minimize the overall opinion of the network. However, link operations for optimizing the overall opinion has not been well studied and understood. In this paper we consider the problem of maximizing the overall opinion by adding edges, namely, making friends in a social network.

We consider a variant of the DeGroot model [29, 30, 42] for opinion dynamics on a social network with $n$ nodes and $m$ edges, where nodes represent individuals, and edges denote interactions among individuals. In this extended DeGroot model, the $n$ individuals are classified two classes $S$ and $F: S$ includes a small number $s \ll n$ of leader nodes, while the remaining $n-s$ nodes in $F$ are followers. In a real social network, a leader could be a paid promoter of a certain product or political position. The leader set $S$ is further categorized into to two subsets $S_{0}$ and $S_{1}$, which represent firm supporters of the opposing parties, containing nodes with opposing opinions 0 and 1 , respectively. In the model, each $i$ node at time $t$ has a nonnegative scalar-value opinion $x_{i}(t)$ in the interval $[0,1]$. When $i$ is a follower, $x_{i}(t)$ evolves as a weighted average of the opinions $x_{j}(t-1)$ of $i$ 's neighbors $j$. When $i$ is a leader, $x_{i}(t)$ never changes over time. Finally, the opinion of each follower converges to a value between 0 and 1 .

We address the following problem based on the aforementioned DeGroot model: Given a social network and a positive integer $k$, how to create $k$ edges, each connecting a 1-valued leader and a follower, so that the overall stead-state opinion is maximized. This problem is at variance with existing work in terms of both formulation and solution. The main contributions of our work are as follows. First, we show that the objective function is monotone and submodular, which are established using a technique completely different from existing ones. Then, we propose two approximation algorithms solving the combinatorial optimization problem based on a greedy strategy of adding edges. The two greedy algorithms are guaranteed to have, respectively, approximation ratios $\left(1-\frac{1}{e}\right)$ and $\left(1-\frac{1}{e}-\epsilon\right)$, where $\epsilon>0$ is the error parameter. The first algorithm has time complexity $O\left(n^{3}\right)$, while the second algorithm has complexity $\widetilde{O}\left(m k \epsilon^{-2}\right)$, where the notation $\widetilde{O}(\cdot)$ hides poly $(\log n)$ 
factors. Finally, comprehensive experiments on various real networks are performed to demonstrate the efficiency and effectiveness of our algorithms, as well as their performance advantages, compared with several baseline strategies of edge addition.

\section{RELATED WORK}

In this section, we briefly review the literature related to our work.

The model under consideration is based on a variant of the popular DeGroot model for opinion dynamics [15]. Since the time the DeGroot model was established, numerous extensions or variants have been proposed by considering different aspects or processes affecting the limiting opinions. For example, the Friedkin-Johnson (FJ) model [18] is a generalization of the DeGroot model, where each individual has two opinions, internal opinion and expressed opinion. After a long time evolution, the expressed opinions of individuals in the FJ model converge but often do not reach agreement. Another extension of the DeGroot model is the Altafini model [3], where it is supposed that the interactions between individuals are not always cooperative, but sometimes antagonistic.

Many existing studies also pay attention to variants of the DeGroot model by selecting 1-leaders in the presence of competing 0-leaders, which are formulated for optimizing different objectives such as minimizing disagreement and polarization [41], maximizing the diversity [30] and the total opinion [29, 31, 42]. Similar opinion maximization problem was also studied for the FJ model by using different strategies, including identifying a given number of 1-leaders [20], as well as modifying individual's internal opinions [39] or susceptibility to persuasion [1,9]. Although the problem we address is also the maximization of the overall opinion, our strategy is optimally selecting edges to add, instead of leader selection.

Admittedly, as a practical approach of graph edit, edge addition operation has been extensively used for different application purposes, such as improving the centrality of a node [12, 13, 37] and maximizing the number of spanning trees [26]. For a social network, creating edges corresponds to making friends. In the field of opinion dynamics, the problem of adding edges has also been studied in order to optimize different objectives. For example, the edge addition strategy was exploited in [7,8], aiming at minimizing the social cost at equilibrium in the FJ model. Again for instance, in [19] and [10], creating edges was adopted to reduce, respectively, controversy and risk of conflict. Finally, edge recommendation was used in [4] with an aim to strategically fight opinion control in a social network. Motivated in part by these work, we exploit the manner of adding edges to maximize the overall opinion. Departing from the literature in the area of opinion dynamics, we present a nearly linear algorithm for evaluating the overall opinion, which is proved to have a guaranteed approximation ratio.

\section{PRELIMINARY}

This section is devoted to a brief introduction to some useful notations and tools, in order to facilitate the description of problem formulation and algorithms.

\subsection{Notations}

We denote scalars in $\mathbb{R}$ by normal lowercase letters like $a, b, c$, sets by normal uppercase letters like $A, B, C$, vectors by bold lowercase letters like $\boldsymbol{a}, \boldsymbol{b}, \boldsymbol{c}$, and matrices by bold uppercase letters like $A, B, C$. We use 1 to denote the vector of appropriate dimensions with all entries being ones, and use $\boldsymbol{e}_{i}$ to denote the $i^{\text {th }}$ standard basis vector of appropriate dimension. Let $\boldsymbol{a}^{\top}$ and $A^{\top}$ denote, respectively, transpose of vector $\boldsymbol{a}$ and matrix $A$. Let $\operatorname{Tr}(A)$ denote the trace of matrix $A$. We use $A_{[i,:]}$ and $A_{[:, j]}$ to denote, respectively, the $i^{\text {th }}$ row and the $j^{\text {th }}$ column of matrix $A$. We write $A_{i, j}$ to denote the entry at row $i$ and column $j$ of $\boldsymbol{A}$ and we use $\boldsymbol{a}_{i}$ to denote the $i^{\text {th }}$ element of vector $\boldsymbol{a}$. We write sets in matrix subscripts to denote submatrices. For example, $\boldsymbol{A}_{F, S}$ represents the submatrix of $A$ with row and column indices in $F$ and $S$, respectively. In addition, we use $A_{F}$ denotes the submatrix of $\boldsymbol{A}$ obtained from $\boldsymbol{A}$ with both the row and column indices in $F$.

For a matrix $X \in \mathbb{R}^{m \times n}$ with entries $X_{i, j}, i=1,2, \cdots, m$ and $j=1,2, \cdots, n$, its Frobenius norm $\|X\|_{F}$ is

$$
\|X\|_{F} \stackrel{\text { def }}{=} \sqrt{\sum_{i=1}^{m} \sum_{j=1}^{n} X_{i, j}^{2}}=\sqrt{\operatorname{Tr}\left(X^{\top} X\right)} .
$$

Definition 3.1. Let $a, b>0$ be positive scalars. $a$ is called an $\epsilon$ approximation $(0<\epsilon<1 / 2)$ of $b$ if the following relation holds: $(1-\epsilon) b \leq a \leq(1+\epsilon) b$, which is denoted $a \approx b$ for simplicity.

For a finite set $X$, let $2^{X}$ be the set of all subsets of $X$. Let $f$ : $2^{X} \rightarrow \mathbb{R}$ be a set function on $2^{X}$. For any subset $T \subseteq W \subseteq X$ and any element $a \in X \backslash W$, the function $f$ is called submodular if $f(T \cup\{a\})-f(T) \geq f(W \cup\{a\})-f(W) . f: 2^{X} \rightarrow \mathbb{R}$ is called monotone increasing if for any subset $T \subseteq W \subseteq X, f(T) \leq f(W)$ holds true.

\subsection{Graphs and Related Matrices}

Let $\mathcal{G}=(V, E)$ be a connected undirected network with $n=|V|$ nodes and $m=|E|$ edges, where $V$ is the set of nodes and $E \subseteq V \times V$ is the set of edges. The adjacency relation of all nodes in $\mathcal{G}$ is encoded in the adjacency matrix $\boldsymbol{A}$, whose entry $\boldsymbol{A}_{i, j}=1$ if $i$ and $i$ are adjacent, and $A_{i, j}=0$ otherwise. Let $\mathcal{N}_{i}$ be the set of neighbouring nodes of $i$. Then degree $d_{i}$ of node $i$ is $\sum_{j=1}^{n} A_{i, j}=\sum_{j \in \mathcal{N}_{i}} A_{i, j}$. The degree diagonal matrix of $\mathcal{G}$ is $D=\operatorname{diag}\left(d_{1}, d_{2}, \cdots, d_{n}\right)$, and the Laplacian matrix $L$ of $G$ is $L=D-A$. If we fix an arbitrary orientation for all edges in $\mathcal{G}$, then we can define the signed edge-node incidence matrix $\boldsymbol{B}_{m \times n}$ of graph $\mathcal{G}$, whose entries are defined as follows: $\boldsymbol{B}_{e, u}=1$ if node $u$ is the head of edge $e, \boldsymbol{B}_{e, u}=-1$ if $u$ is the tail of $e$, and $B_{e, u}=0$ otherwise. For an oriented edge $e \in E$ with end nodes $u$ and $v$, we define $\boldsymbol{b}_{\boldsymbol{e}}=\boldsymbol{b}_{u, v}=\boldsymbol{e}_{u}-\boldsymbol{e}_{v}$ if $u$ and $v$ are, respectively, the head and tail of $e$. Then $L$ can be written as $\boldsymbol{L}=\boldsymbol{B}^{\top} \boldsymbol{B}$ or $\boldsymbol{L}=\sum_{\boldsymbol{e} \in E} \boldsymbol{b}_{\boldsymbol{e}} \boldsymbol{b}_{\boldsymbol{e}}^{\top}$, meaning that $\boldsymbol{L}$ is positive semidefinite. Moreover, for a nonnegative diagonal matrix $X$ with at least one nonzero diagonal entry, we have the fact that every element of $(L+X)^{-1}$ is positive [27, 31, 32].

\section{PROBLEM FORMULATION}

In this section, we formulate the problem for optimizing the opinion influence of leaders in a social network described by a graph 
$\mathcal{G}(V, E)$, where nodes represent individuals or agents, and edges represent interactions among agents. We adopt the discrete-time leader-follower DeGroot model [29, 30, 42] for opinion dynamics to achieve our goal.

\subsection{Leader-Follower DeGroot Model}

In the leader-follower DeGroot model on graph $\mathcal{G}$, node set is divided into two disjoint parts, a set $S$ of a small number $s \ll n$ leader nodes and a follower set $F$ containing the remaining $n-s$ nodes, where $S$ is the union of two disjoint subsets $S_{0}$ and $S_{1}$ presenting two competing parties and satisfying $S=S_{0} \cup S_{1}$. Each node has a nonnegative scalar-value opinion belongs to interval $[0,1]$. Let $x_{i}(t)$ be the opinion of node $i$ at time $t$. If $i \in S_{0}, x_{i}(t)$ keeps unchanged, meaning $x_{i}(t)=0$ for all $t$; if $i \in S_{1}, x_{i}(t)=1$ for all $t$. For a follower node $i \in F$ with an initial opinion $x_{i}(0)$, it will update its opinion by averaging all its neighbours' opinions as

$$
x_{i}(t+1)=\frac{\sum_{j \in \mathcal{N}_{i}} A_{i, j} x_{j}(t)}{\sum_{j \in \mathcal{N}_{i}} A_{i, j}} .
$$

Let $x_{F}(t)$ and $x_{S}(t)$ be, respectively, opinion vectors of followers and leaders at time $t$. For large $t, x_{F}(t)$ and $x_{S}(t)$ converge. Let $x_{S}(\infty)=\lim _{t \rightarrow \infty} x_{S}(t)$ and $x_{F}(\infty)=\lim _{t \rightarrow \infty} x_{F}(t)$. We write matrices $A$ and $L$ in block form as

$$
\boldsymbol{A}=\left(\begin{array}{ll}
\boldsymbol{A}_{S, S} & \boldsymbol{A}_{S, F} \\
\boldsymbol{A}_{F, S} & \boldsymbol{A}_{F, F}
\end{array}\right), \boldsymbol{L}=\left(\begin{array}{ll}
\boldsymbol{L}_{S, S} & \boldsymbol{L}_{S, F} \\
\boldsymbol{L}_{F, S} & \boldsymbol{L}_{F, F}
\end{array}\right) .
$$

Then, the stationary opinions can be determined as follows [40]:

$$
\begin{aligned}
& x_{S}(\infty)=x_{S}(0), \\
& x_{F}(\infty)=L_{F, F}^{-1} A_{F, S} x_{S}(0)=L_{F}^{-1} A_{F, S} x_{S}(0) .
\end{aligned}
$$

\subsection{Problem Statement}

Equation (3) shows that for any follower node, its equilibrium opinion is determined by the opinions of leaders, independent of its own initial opinion. Thus, the leaders affect the opinions of followers. Let $H(\mathcal{G})$ denote the sum of stable opinions over all followers in graph $\mathcal{G}$, given by $H(\mathcal{G})=\sum_{i \in F} \boldsymbol{x}_{i}(\infty)=\mathbf{1}^{\top} \boldsymbol{L}_{F}^{-1} \boldsymbol{A}_{F, S} \boldsymbol{x}_{S}(0)$, which encodes the influence of leaders on the opinions of followers. In the sequel, by using the quantity $H(\mathcal{G})$, we formulate the problem for maximizing the influence of the 1-leader by adding a fixed number of edges from a candidate set of edges. For simplicity, we define a vector $\boldsymbol{b}=\boldsymbol{A}_{F, S} \boldsymbol{x}_{S}(0)$. By definition, for each node $i \in F, b_{i}$ is in fact equal to the number of edges connecting node $i$ and nodes in set $S_{1}$. Then, the vector for equilibrium opinions of followers can be simplified as $\boldsymbol{x}_{F}(\infty)=\boldsymbol{L}_{F}^{-1} \boldsymbol{b}$ and $H(\mathcal{G})$ can be rewritten as $H(\mathcal{G})=\mathbf{1}^{\top} \boldsymbol{L}_{F}^{-1} \boldsymbol{b}$.

For a connected undirected graph $\mathcal{G}(V, E)$, if we add a set $T$ of nonexistent edges from a candidate set $Q$ to $\mathcal{G}$ forming a new graph $\mathcal{G}(T)=(V, E \cup T)$, where each new edge connects a 1-leader node in $S_{1}$ and a follower node in $F$, the overall equilibrium opinion of follower nodes in $\mathcal{G}(T)=(V, E \cup T)$ will increase. We next prove this property. For simplicity, we simplify $H(G(T))$ as $H(T)$, which means $H(\mathcal{G})=H(\emptyset)$. Then, we have the following result.

LEMma 4.1. Let $\mathcal{G}=(V, E)$ be a connected graph with nonempty follower set F, 0-leader set $S_{0}$, and 1-leader set $S_{1}$. Let e $\notin E$ be a potential edge incident to node $i \in F$ and a 1-leader in $S_{1}$. Define

$$
\begin{aligned}
\Delta(e) \stackrel{\text { def }}{=} H(\{e\})-H(\emptyset) & \text { Then, } \\
\Delta(e) & =\frac{\mathbf{1}^{\top} \boldsymbol{L}_{F}^{-1} \boldsymbol{e}_{i}\left(1-\boldsymbol{e}_{i}^{\top} \boldsymbol{L}_{F}^{-1} \boldsymbol{b}\right)}{1+\boldsymbol{e}_{i}^{\top} \boldsymbol{L}_{F}^{-1} \boldsymbol{e}_{i}}
\end{aligned}
$$

and $\Delta(e) \geq 0$.

Proof. By definition, one obtains $H(\{e\})=H(G(\{e\}))=\mathbf{1}^{\top}\left(L_{F}+\right.$ $\left.\boldsymbol{e}_{i} \boldsymbol{e}_{i}^{\top}\right)^{-1}\left(\boldsymbol{b}+\boldsymbol{e}_{i}\right)$. Exploiting Sherman-Morrison formula [33], it follows that

$$
\left(L_{F}+\boldsymbol{e}_{i} \boldsymbol{e}_{i}^{\top}\right)^{-1}=L_{F}^{-1}-\frac{\boldsymbol{L}_{F}^{-1} \boldsymbol{e}_{i} \boldsymbol{e}_{i}^{\top} \boldsymbol{L}_{F}^{-1}}{1+\boldsymbol{e}_{i}^{\top} \boldsymbol{L}_{F}^{-1} \boldsymbol{e}_{i}} .
$$

Then, $\Delta(e)$ is evaluated as

$$
\begin{aligned}
\Delta(e) & =\mathbf{1}^{\top}\left(\boldsymbol{L}_{F}^{-1}-\frac{\boldsymbol{L}_{F}^{-1} \boldsymbol{e}_{i} \boldsymbol{e}_{i}^{\top} \boldsymbol{L}_{F}^{-1}}{1+\boldsymbol{e}_{i}^{\top} \boldsymbol{L}_{F}^{-1} \boldsymbol{e}_{i}}\right)\left(\boldsymbol{b}+\boldsymbol{e}_{i}\right)-\mathbf{1}^{\top} \boldsymbol{L}_{F}^{-1} \boldsymbol{b} \\
& =\frac{\mathbf{1}^{\top} \boldsymbol{L}_{F}^{-1} \boldsymbol{e}_{i}\left(1-\boldsymbol{e}_{i}^{\top} \boldsymbol{L}_{F}^{-1} b\right)}{1+\boldsymbol{e}_{i}^{\top} \boldsymbol{L}_{F}^{-1} \boldsymbol{e}_{i}} .
\end{aligned}
$$

Notice that $\boldsymbol{e}_{i}^{\top} \boldsymbol{L}_{F}^{-1} \boldsymbol{b}$ is the equilibrium opinion of follower node $i$ in graph $\mathcal{G}$, which is no more than 1 . On the other hand, any entry of matrix $L_{F}^{-1}$ is nonnegative. Hence, $\Delta(e) \geq 0$.

Lemma 4.1 indicates that the addition of any nonexisting edge connecting a 1-leader and a follower will lead to an increase of the overall equilibrium opinion of followers. Then we naturally raise the following problem called OpinionMaximization: How to optimally select a set $T$ with $k$ edge in a candidate edge set $Q$, so that the influence of 1-leaders quantified by the overall opinion of leaders in the new graph is maximized. Mathematically, the influence maximization problem can be formally stated as follows.

Problem 1 (Opinion Maximization). Given a connected undirected graph $\mathcal{G}=(V, E)$, a nonempty set $S_{0}$ of 0-valued leaders, a set $S_{1} \neq \emptyset$ of 1-leaders, a nonempty set $F=V \backslash\left(S_{0} \cup S_{1}\right)$ of followers, the candidate edge set $Q \subseteq F \times S_{1}$ consisting of nonexistent edges connecting 1-leaders and follower nodes, and an integer $k$, we aim to find the edge set $T \subseteq Q$ with $|T|=k$, and add these chosen $k$ edges to graph $\mathcal{G}$ forming a new graph $\mathcal{G}(T)=(V, E \cup T)$, so that the overall opinion $H(T)$ is maximized. This set optimization problem can be formulated as:

$$
T=\arg \max _{P \subseteq Q,|P|=k} H(P) .
$$

Similarly, we can maximize the influence of 0 -valued leaders by adding edges to graph $\mathcal{G}$ to minimize the overall opinion of followers, which is called OpinionMinimization problem. Since for both problems, the proof and algorithms are similar, in what follows, we only consider the OpinionMaximization problem.

Note that given set $S_{1}$ of 1-leaders, the number of absent edges connecting 1-leaders and followers in a sparse network is large with order $O\left(\left|S_{1}\right| n\right)$. However, if the stationary opinion of follower node $i$ is close to 1 , the benefit of adding a new edge between $i$ and a node in set $S_{1}$ is relatively low. Thus, we will not inspect all of $O\left(\left|S_{1}\right| n\right)$ nonexistent edges, but focus on a small number of candidate edges with $|Q| \ll\left|S_{1}\right| n$. Specifically, we set a threshold $\eta(1 / 2<\eta<1)$ and determine the candidate set $Q$ according to the following rule. For each follower node $i$ not adjacent to any 1leader in graph $\mathcal{G}$, if $x_{i}(\infty)<\eta, Q$ includes the nonexistent edges connecting $i$ and 1-leaders. Similar restriction to the candidate set 
$Q$ of edges is previously used in [4], where the edges in $Q$ are called "good" candidate edges.

\subsection{Properties of Objective Function}

Here we prove that as the objective function of Problem 1, the set function $H(\cdot)$ has two desirable properties: monotonicity and submodularity. First, by Lemma 4.1, it is immediate that function $H(\cdot)$ is monotonically increasing.

Theorem 4.2. (Monotonicity) For two subsets $B$ and $T$ of edges satisfying $B \subset T \subset Q, H(B) \leq H(T)$ holds.

Next, we show that function $H(\cdot)$ is submodular.

Theorem 4.3. (Submodularity) For two subsets $B$ and $T$ obeying $B \subset T \subseteq Q$ and any edge $e \in Q \backslash T$,

$$
H(T \cup\{e\})-H(T) \leq H(B \cup\{e\})-H(B) .
$$

Proof. Let $e_{1}=(a, i)$ and $e_{2}=(b, j)$ be two edges in $Q$ with $a, b \in S_{1}$ and $i, j \in F$. We now prove that for this simple case, one has

$$
H\left(\left\{e_{1} \cup e_{2}\right\}\right)-H\left(\left\{e_{1}\right\}\right) \leq H\left(\left\{e_{2}\right\}\right)-H(\emptyset) .
$$

To this end, we define matrix $\Omega(x, y)=\left(\boldsymbol{L}_{F}+x \boldsymbol{E}_{i i}+y \boldsymbol{E}_{j j}\right)^{-1}$ and vector $\bar{b}(x, y)=\boldsymbol{b}+x \boldsymbol{e}_{i}+y \boldsymbol{e}_{j}$, where $\boldsymbol{E}_{i i}=\boldsymbol{e}_{i} \boldsymbol{e}_{i}^{\top}, x \geq 0$ and $y \geq 0$. By definition, it is easy to verify that the entries in $\Omega(x, y)$ are nonnegative, and inequality (7) can be rephrased as

$$
\begin{aligned}
& \mathbf{1}^{\top} \Omega(1,1) \bar{b}(1,1)-\mathbf{1}^{\top} \Omega(0,1) \bar{b}(0,1) \\
\leq & \mathbf{1}^{\top} \Omega(1,0) \bar{b}(1,0)-\mathbf{1}^{\top} \Omega(0,0) \bar{b}(0,0) .
\end{aligned}
$$

In order to prove (8), we introduce a function $f(x, y) \stackrel{\text { def }}{=} \mathbf{1}^{\top} \Omega(x, y)$ $\bar{b}(x, y)$. Next we prove

$$
f(x, y)-f(0, y) \leq f(x, 0)-f(0,0)
$$

the special case $x=y=1$ of which is exactly (8). In order to prove (9), it suffices to prove $f_{x y}(x, y) \leq 0$, the proof of which involves the following matrix derivative formula

$$
\frac{d}{d t} A(t)^{-1}=-A(t)^{-1} \frac{d}{d t} A(t) A(t)^{-1}
$$

Since $H(\cdot)$ is a monotone increasing function, we have

$$
\begin{aligned}
& f_{x}(x, y)=-\mathbf{1}^{\top} \Omega(x, y) \boldsymbol{E}_{i i} \Omega(x, y) \bar{b}(x, y)+\mathbf{1}^{\top} \Omega(x, y) \boldsymbol{e}_{i} \geq 0, \\
& f_{y}(x, y)=-\boldsymbol{1}^{\top} \Omega(x, y) E_{j j} \Omega(x, y) \bar{b}(x, y)+\boldsymbol{1}^{\top} \Omega(x, y) \boldsymbol{e}_{j} \geq 0 .
\end{aligned}
$$

Then, $f_{x y}(x, y)$ can be computed as

$$
\begin{aligned}
f_{x y}(x, y)= & \mathbf{1}^{\top} \Omega(x, y) \boldsymbol{E}_{j j} \Omega(x, y) \boldsymbol{E}_{i i} \Omega(x, y) \overline{\boldsymbol{b}}(x, y)+ \\
& \mathbf{1}^{\top} \Omega(x, y) \boldsymbol{E}_{i i} \Omega(x, y) \boldsymbol{E}_{j j} \Omega(x, y) \bar{b}(x, y)- \\
& \mathbf{1}^{\top} \Omega(x, y) \boldsymbol{E}_{i i} \Omega(x, y) \boldsymbol{e}_{j}-\mathbf{1}^{\top} \Omega(x, y) \boldsymbol{E}_{j j} \Omega(x, y) \boldsymbol{e}_{i} \\
= & -\boldsymbol{e}_{i}^{\top} \Omega(x, y) \boldsymbol{e}_{j}\left(f_{x}(x, y)+f_{y}(x, y)\right) \leq 0 .
\end{aligned}
$$

Combining (8), (9) and (10) yields (7).

We assume $|T \backslash B|=z$ and $T \backslash B=\left\{e_{1}, e_{2}, \ldots, e_{z}\right\}$, and define a graph sequence $\mathcal{G}^{(i)}(i=0,1,2, \ldots, z)$ with identical node set $V$ but different edge set $E^{(i)}$, obeying $\mathcal{G}^{(0)}=\mathcal{G}, E^{(0)}=E$, and $E^{(i)} \backslash E^{(i-1)}=e_{i}$. By iteratively applying (7) to $\mathcal{G}^{(i)}$ leads to (6), we can conclude the proof of submodularity.

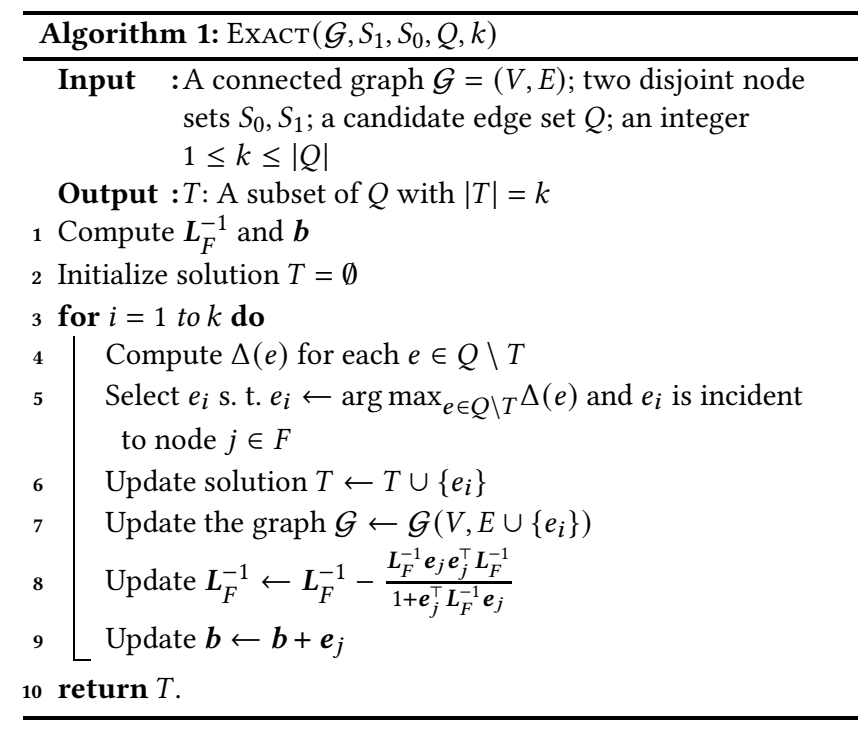

\section{SIMPLE GREEDY ALGORITHM}

Problem 1 is inherently a combinatorial problem. It can be solved by the following naïve brute-force approach. For each set $T$ of the $\left(\begin{array}{c}|Q| \\ k\end{array}\right)$ possible subsets of edges, compute the overall equilibrium opinion of follower nodes in the resultant graph when all edges in this set are added. Then, output the subset $T^{*}$ of edges, whose addition leads to the largest increase for the overall opinion of followers. Although this method is simple, it is computationally impossible even for small networks, since its has an exponential complexity $O\left(\left(\begin{array}{c}|Q| \\ k\end{array}\right) \cdot(n-s)^{3}\right)$.

To tackle the exponential complexity, one often resorts to greedy heuristics. Due to the monotonicity and submodularity of the objective function $H(\cdot)$, a simple greedy strategy is guaranteed to have a $\left(1-\frac{1}{e}\right)$-approximation solution to Problem 1 [35]. Initially, we set the edge set $T$ to be empty, then $k$ edges are added from set $Q \backslash T$ iteratively. In each iteration step $i$, edge $e_{i}$ in set $Q$ of candidate edges is selected, which maximizes the overall opinion of followers. The algorithm terminates when $k$ edges are selected to be added to $T$. For every candidate edge, it needs computation of the overall opinion, which involves matrix inversion. A direct calculation of matrix inversion requires $O\left((n-s)^{3}\right)$ time, leading to a total computation complexity $O\left(k|Q|(n-s)^{3}\right)$.

Actually, as shown in the proof of Lemma 4.1, if $L_{F}^{-1}$ is already computed, adding a single edge can be looked upon as a rank-1 update to matrix $L_{F}^{-1}$, which can be done in time $O\left((n-s)^{2}\right)$ by using the Sherman-Morrison formula [33], rather than directly inverting a matrix in time $O\left((n-s)^{3}\right)$. This leads to our simple algorithm $\operatorname{ExAct}\left(G, S_{1}, S_{0}, Q, k\right)$ to solve Problem 1 , which is outlined in Algorithm 1 . The algorithm first calculates the inversion of matrix $L_{F}$ in time $O\left((n-s)^{3}\right)$. Then it works in $k$ rounds with each round containing two main operations. One is to compute $\Delta(e)$ (Line 4$)$ in $O(n(n-s))$ time, the other is to update $L_{F}^{-1}$ in $O\left((n-s)^{2}\right)$ time when a new edge $e_{i}$ (Line 8$)$ is added. Therefore, the whole running time of Algorithm 1 is $O\left((n-s)^{3}+k n(n-s)\right)$, much faster than the brute-force algorithm. 
Based on the well-established result [35], Algorithm 1 yields a ( $1-\frac{1}{e}$ )-approximation of the optimal solution to Problem 1, as provided in the following theorem.

Theorem 5.1. The set $T$ returned by Algorithm 1 satisfies

$$
H(T)-H(\emptyset) \geq\left(1-\frac{1}{e}\right)\left(H\left(T^{*}\right)-H(\emptyset)\right)
$$

where $T^{*}$ is the optimal solution to Problem 1 satisfying

$$
H\left(T^{*}\right)=\underset{P \subset Q,|P|=k}{\arg \max } H(P) .
$$

\section{FAST GREEDY ALGORITHM}

Although the computation complexity of Algorithm 1 is much lower than the brute-force algorithm, it still cannot handle large-scale networks since it requires inverting matrix $L_{F}$ in cube time. As shown above, the key step to solve Problem 1 is to compute the quantity $\Delta(e)$ given in (4), which is the increment of overall opinion resulted by the addition of edge $e$. In this section, we provide efficient approximations for the three terms $\mathbf{1}^{\top} \boldsymbol{L}_{F}^{-1} \boldsymbol{e}_{i}, 1-\boldsymbol{e}_{i}^{\top} \boldsymbol{L}_{F}^{-1} \boldsymbol{b}$, and $\boldsymbol{e}_{i}^{\top} \boldsymbol{L}_{F}^{-1} \boldsymbol{e}_{i}$ in the numerator and denominator of (4), which avoid inverting a matrix. These approximations together lead to an errorguaranteed approximation to $\Delta(e)$ and thus a fast approximation algorithm to Problem 1 that has $\left(1-\frac{1}{e}-\epsilon\right)$ approximation ratio and time complexity $\tilde{O}\left(k m \epsilon^{-2}\right)$ for any error parameter $0<\epsilon<1 / 2$.

In order to reduce computational cost, it is important to avoid the inversion of matrix $L_{F}$, which can be done since matrix $L_{F}$ has many desirable properties as will be shown below. For example, it can be expressed as the sum of a Laplacian matrix associated with a graph and a nonnegative diagonal matrix. For a connected graph $\mathcal{G}=(V, E)$ with a subset $F \subset V$ of follower nodes and a subset $S \subset V$ of leader nodes, we define a graph $\overline{\mathcal{G}}=(F,(F \times F) \cap E)$ with $n-s$ nodes and $\bar{m}$ edges, which is a subgraph of $\mathcal{G}$. Let $\bar{B}$ be the incidence matrix of $\overline{\mathcal{G}}$ and $\overline{\boldsymbol{L}}$ be its Laplacian matrix. Then, matrix $\boldsymbol{L}_{F}$ can be represented as $\boldsymbol{L}_{F}=\overline{\boldsymbol{B}}^{\top} \overline{\boldsymbol{B}}+\boldsymbol{W}$, where $\boldsymbol{W}$ is a nonnegative diagonal matrix with the $i^{\text {th }}$ diagonal entry equal to the number of edges connecting the follower node $i$ and nodes in $S$. Thus, $\boldsymbol{L}_{F}$ is a symmetric, diagonally-dominant M-matrix (SDDM). Moreover, it is easy to obtain that $\boldsymbol{e}_{i}^{\top} \boldsymbol{L}_{F}^{-1} \boldsymbol{e}_{i} \geq 1 / n$ and $\operatorname{Tr}\left(\boldsymbol{L}_{F}^{-1}\right) \leq$ $\operatorname{Tr}\left(\overline{\boldsymbol{L}}^{-1}\right) \leq\left(n^{2}-1\right) / 6$ [28]. These properties are helpful for the following derivations.

In addition, the following lemma is also instrumental for approximating the related quantities.

LEMma 6.1. For an arbitrary vector $v$ of $n-s$ dimension, $v_{i}^{2} \leq$ $n\|\boldsymbol{v}\|_{\boldsymbol{L}_{F}}^{2}$ holds, where $\|\boldsymbol{v}\|_{L_{F}}^{2}=\boldsymbol{v}^{\top} \boldsymbol{L}_{F} \boldsymbol{v}$.

Proof. Considering $\boldsymbol{L}_{F}=\overline{\boldsymbol{B}}^{\top} \overline{\boldsymbol{B}}+\boldsymbol{W}$, we distinguish two cases: $W_{i, i} \geq 1$ and $W_{i, i}=0$. For the first case $W_{i, i} \geq 1$, it is apparent that $v_{i}^{2} \leq n\|v\|_{L_{F}}^{2}$. While for the second case $W_{i, i}=0$, there exists a follower node $j$ with corresponding element $W_{j, j} \geq 1$ in the component of graph $\bar{G}$ that contains node $j$. Let $P_{i j}$ be a simple path connecting node $i$ and $j$ in graph $\overline{\mathcal{G}}$, the length of which is at most $n$. Then, we have

$$
\begin{aligned}
\|\boldsymbol{v}\|_{L_{F}}^{2} & \geq \sum_{(a, b) \in P_{i j}}\left(\boldsymbol{v}_{a}-\boldsymbol{v}_{b}\right)^{2}+\boldsymbol{v}_{j}^{2} \\
& \geq \frac{\left(\sum_{(a, b) \in P_{i j}}\left(\boldsymbol{v}_{a}-\boldsymbol{v}_{b}\right)+\boldsymbol{v}_{j}\right)^{2}}{n} \geq \frac{\boldsymbol{v}_{i}^{2}}{n},
\end{aligned}
$$

which completes the proof.

\subsection{Approximations of Numerator in (4)}

We now approximate the two terms $\mathbf{1}^{\top} \boldsymbol{L}_{F}^{-1} \boldsymbol{e}_{i}, 1-\boldsymbol{e}_{i}^{\top} \boldsymbol{L}_{F}^{-1} \boldsymbol{b}$ in the numerator in (4). Since $L_{F}$ is a SDDM matrix, one can resort to the fast symmetric, diagonally-dominant (SDD) linear system solver [11, 38] to evaluate $\mathbf{1}^{\top} \boldsymbol{L}_{F}^{-1} \boldsymbol{e}_{i}$ and $1-\boldsymbol{e}_{i}^{\top} \boldsymbol{L}_{F}^{-1} \boldsymbol{b}$, which avoids inverting matrix $\boldsymbol{L}_{F}$.

LEMMA 6.2. There is a nearly linear time solver $x=\operatorname{SOLVE}(S, y, \epsilon)$ which takes a symmetric positive semi-definite matrix $S_{n \times n}$ with $m$ nonzero entries, a vector $\boldsymbol{b} \in \mathbb{R}^{n}$, and an error parameter $\delta>0$, and returns a vector $x \in \mathbb{R}^{n}$ satisfying $\left\|x-S^{-1} y\right\|_{S} \leq \delta\left\|S^{-1} y\right\|_{S}$ with high probability, where $\|x\|_{S} \stackrel{\text { def }}{=} \sqrt{x^{\top} S x}$. The solver runs in expected time $\tilde{O}(m)$, where $\tilde{O}(\cdot)$ notation suppresses the poly $(\log n)$ factors.

Based on this solver, $\mathbf{1}^{\top} \boldsymbol{L}_{F}^{-1} \boldsymbol{e}_{i}, 1-\boldsymbol{e}_{i}^{\top} \boldsymbol{L}_{F}^{-1} \boldsymbol{b}$ are approximated in Lemmas 6.3 and 6.4, respectively.

Lemma 6.3. Given an undirected unweighted graph $\mathcal{G}=(V, E)$, the matrix $\boldsymbol{L}_{F}$ and a parameter $0<\epsilon<1 / 2$, let $\boldsymbol{h}=\operatorname{SOLVE}\left(\boldsymbol{L}_{F}, \mathbf{1}, \delta_{1}\right)$ where $\delta_{1}=\frac{\epsilon}{2 n^{2} \sqrt{6\left(n^{2}-1\right)}}$. Then for any $i \in F$, we have

$$
\mathbf{1}^{\top} \boldsymbol{L}_{F}^{-1} \boldsymbol{e}_{i} \stackrel{\epsilon / 6}{\approx} \boldsymbol{h}_{i}
$$

Proof. Define $\tilde{\boldsymbol{h}}=\boldsymbol{L}_{F}^{-1} \mathbf{1}$, then $\tilde{\boldsymbol{h}}_{i}=\mathbf{1}^{\top} \boldsymbol{L}_{F}^{-1} \boldsymbol{e}_{i}$. According to Lemmas 6.1 and 6.2, one obtains

$$
\begin{aligned}
\left(\boldsymbol{h}_{i}-\tilde{\boldsymbol{h}}_{i}\right)^{2} & \leq n\|\boldsymbol{h}-\tilde{\boldsymbol{h}}\|_{\boldsymbol{L}_{F}}^{2} \leq \delta_{1}^{2} n\|\tilde{\boldsymbol{h}}\|_{\boldsymbol{L}_{F}}^{2} \\
& \leq \delta_{1}^{2} n^{2} \operatorname{Tr}\left(\boldsymbol{L}_{F}^{-1}\right) \leq \delta_{1}^{2} n^{2}\left(n^{2}-1\right) / 6 .
\end{aligned}
$$

On the other hand, $\tilde{\boldsymbol{h}}_{\boldsymbol{i}}$ can be bounded as

$$
\tilde{\boldsymbol{h}}_{i}=\mathbf{1}^{\top} \boldsymbol{L}_{F}^{-1} \boldsymbol{e}_{i} \geq \boldsymbol{e}_{i}^{\top} \boldsymbol{L}_{F}^{-1} \boldsymbol{e}_{i} \geq \frac{1}{2 n}
$$

Then, one has

$$
\frac{\left|\boldsymbol{h}_{i}-\tilde{\boldsymbol{h}}_{i}\right|}{\tilde{\boldsymbol{h}}_{i}} \leq 2 \delta_{1} n^{2} \sqrt{\left(n^{2}-1\right) / 6} \leq \frac{\epsilon}{6},
$$

which completes the proof.

Lemma 6.4. Given an undirected unweighted graph $\mathcal{G}=(V, E)$, the matrix $L_{F}$ and two parameters $0<\epsilon<1 / 2,1 / 2<\eta<1$, let $\boldsymbol{p}=\operatorname{SOLVE}\left(\boldsymbol{L}_{F}, \boldsymbol{b}, \delta_{2}\right)$ where $\delta_{2}=\frac{(1-\eta) \epsilon}{n^{2} \sqrt{6\left(n^{2}-1\right)}}$. Then for any $i \in F$, we have

$$
1-\boldsymbol{e}_{i}^{\top} \boldsymbol{L}_{F}^{-1} \boldsymbol{b} \stackrel{\epsilon / 6}{\approx} 1-\boldsymbol{p}_{i}
$$

Proof. Define $\tilde{\boldsymbol{p}}=\boldsymbol{L}_{F}^{-1} \boldsymbol{b}$, then $\tilde{\boldsymbol{p}}_{i}=\boldsymbol{e}_{i}^{\top} \boldsymbol{L}_{F}^{-1} \boldsymbol{b}$. Considering the restriction of the set $Q$ of candidate edges, for each follower $i$ connecting by an $e \in Q, \boldsymbol{e}_{i}^{\top} \boldsymbol{L}_{F}^{-1} \boldsymbol{b} \leq \eta$ holds. By Lemmas 6.1 and 6.2, we have

$$
\left|\left(1-\tilde{\boldsymbol{p}}_{i}\right)-\left(1-\boldsymbol{p}_{i}\right)\right|=\left|\tilde{\boldsymbol{p}}_{i}-\boldsymbol{p}_{i}\right| \leq \delta_{2} n^{2} \sqrt{\left(n^{2}-1\right) / 6} .
$$


Therefore,

$$
\frac{\left|\left(1-\boldsymbol{p}_{i}\right)-\left(1-\tilde{\boldsymbol{p}}_{i}\right)\right|}{1-\tilde{\boldsymbol{p}}_{i}} \leq \frac{\delta_{2} n^{2} \sqrt{\left(n^{2}-1\right) / 6}}{1-\eta} \leq \frac{\epsilon}{6},
$$

which finishes the proof.

\subsection{Approximation of Denominator (4)}

Using the expression $\boldsymbol{L}_{F}=\overline{\boldsymbol{B}}^{\top} \overline{\boldsymbol{B}}+\boldsymbol{W}, \boldsymbol{e}_{i}^{\top} \boldsymbol{L}_{F}^{-1} \boldsymbol{e}_{i}$ is recast as

$$
\begin{aligned}
& \boldsymbol{e}_{i}^{\top} \boldsymbol{L}_{F}^{-1} \boldsymbol{e}_{i}=\boldsymbol{e}_{i}^{\top} \boldsymbol{L}_{F}^{-1}\left(\overline{\boldsymbol{B}}^{\top} \overline{\boldsymbol{B}}+\boldsymbol{W}\right) \boldsymbol{L}_{F}^{-1} \boldsymbol{e}_{i} \\
= & \boldsymbol{e}_{i}^{\top} \boldsymbol{L}_{F}^{-1} \overline{\boldsymbol{B}}^{\top} \overline{\boldsymbol{B}} \boldsymbol{L}_{F}^{-1} \boldsymbol{e}_{i}+\boldsymbol{e}_{i}^{\top} \boldsymbol{L}_{F}^{-1} \boldsymbol{W} \boldsymbol{L}_{F}^{-1} \boldsymbol{e}_{i} \\
= & \left\|\overline{\boldsymbol{B}} \boldsymbol{L}_{F}^{-1} \boldsymbol{e}_{i}\right\|^{2}+\left\|\boldsymbol{W}^{\frac{1}{2}} \boldsymbol{L}_{F}^{-1} \boldsymbol{e}_{i}\right\|^{2} .
\end{aligned}
$$

In this way, we have reduced the estimation of $\boldsymbol{e}_{i}^{\top} \boldsymbol{L}_{F}^{-1} \boldsymbol{e}_{i}$ to the calculation of the $\ell_{2}$ norms $\left\|\bar{B} L_{F}^{-1} \boldsymbol{e}_{i}\right\|^{2}$ and $\left\|\boldsymbol{W}^{\frac{1}{2}} \boldsymbol{L}_{F}^{-1} \boldsymbol{e}_{i}\right\|^{2}$ of two vectors in $\mathbb{R}^{\bar{m}}$ and $\mathbb{R}^{n-s}$, respectively. However, the complexity for exactly computing these two $\ell_{2}$ norms is still high. In order to alleviate the computation burden, we apply the Johnson-Lindenstrauss (JL) Lemma $[2,22]$ to approximate the $\ell_{2}$ norms. For example, for $\left\|\bar{B} L_{F}^{-1} \boldsymbol{e}_{i}\right\|^{2}$, if we project a set of $n-s$ vectors of $\bar{m}$ dimension (like the columns of matrix $\bar{B} L_{F}^{-1}$ ) onto a low $t$-dimensional subspace spanned by the columns of a random matrix $Q \in \mathbb{R}^{t \times \bar{m}}$ with entries being $\pm 1 / \sqrt{t}$, where $t=\left\lceil 24 \log (n-s) / \epsilon^{2}\right\rceil$ for given $\epsilon$, then we get an $\epsilon$-approximation of $\left\|\bar{B} L_{F}^{-1} \boldsymbol{e}_{i}\right\|^{2}$ with high probability. This $\ell_{2}$ norm preserving projection method is also applicable to the estimation of $\left\|\boldsymbol{W}^{\frac{1}{2}} \boldsymbol{L}_{F}^{-1} \boldsymbol{e}_{i}\right\|^{2}$. For consistency, we introduce the JL Lemma [2, 22].

Lemma 6.5. Given fixed vectors $\boldsymbol{v}_{1}, \boldsymbol{v}_{2}, \ldots, \boldsymbol{v}_{n} \in \mathbb{R}^{d}$ and $\epsilon>0$, let $Q_{t \times d}, t \geq 24 \log n / \epsilon^{2}$, be a matrix, each entry of which is equal to $1 / \sqrt{t}$ or $-1 / \sqrt{t}$ with the same probability $1 / 2$. Then with probability at least $1-1 / n$,

$$
(1-\epsilon)\left\|v_{i}-v_{j}\right\|^{2} \leq\left\|Q v_{i}-Q v_{j}\right\|^{2} \leq(1+\epsilon)\left\|v_{i}-v_{j}\right\|^{2}
$$

for all pairs $i, j \leq n$.

Let $Q_{t \times \bar{m}}$ and $\boldsymbol{P}_{t \times(n-s)}$ be two random $\pm 1 / \sqrt{t}$ matrices where $t=\left\lceil 24 \log (n-s) / \epsilon^{2}\right\rceil$. By Lemma 6.5, for any $i \in F$ we have

$$
\boldsymbol{e}_{i}^{\top} \boldsymbol{L}_{F}^{-1} \boldsymbol{e}_{i} \stackrel{\epsilon}{\approx}\left\|Q \bar{B} L_{F}^{-1} \boldsymbol{e}_{i}\right\|^{2}+\left\|P W^{\frac{1}{2}} L_{F}^{-1} \boldsymbol{e}_{i}\right\|^{2}
$$

Let $\bar{X}=Q \bar{B}, X=\bar{B} L_{F}^{-1}, X^{\prime}=Q \bar{B} L_{F}^{-1}, \tilde{X}_{[j,:]}=\operatorname{Solve}\left(L_{F}, \bar{X}_{[j,:]}, \delta\right)$ and $\bar{Y}=\boldsymbol{P} \boldsymbol{W}^{\frac{1}{2}}, \boldsymbol{Y}=\boldsymbol{W}^{\frac{1}{2}} \boldsymbol{L}_{F}^{-1}, \boldsymbol{Y}^{\prime}=\boldsymbol{P} \boldsymbol{W}^{\frac{1}{2}} \boldsymbol{L}_{F}^{-1}, \tilde{\boldsymbol{Y}}_{[j,:]}=\operatorname{Solve}\left(\boldsymbol{L}_{F}, \bar{Y}_{[j,:]}\right.$, $\delta$ ). Then, $\boldsymbol{e}_{i}^{\top} \boldsymbol{L}_{F}^{-1} \boldsymbol{e}_{i}=\left\|\boldsymbol{X} \boldsymbol{e}_{i}\right\|^{2}+\left\|Y \boldsymbol{e}_{i}\right\|^{2}$. Combining Lemmas 6.2 and 6.5, we get an approximation of $\boldsymbol{e}_{i}^{\top} \boldsymbol{L}_{F}^{-1} \boldsymbol{e}_{i}$.

LEMma 6.6. Suppose that $\forall i \in F$,

$$
\begin{aligned}
& (1-\epsilon / 12)\left\|X \boldsymbol{e}_{i}\right\|^{2} \leq\left\|X^{\prime} \boldsymbol{e}_{i}\right\|^{2} \leq(1+\epsilon / 12)\left\|X \boldsymbol{e}_{i}\right\|^{2}, \\
& (1-\epsilon / 12)\left\|Y \boldsymbol{e}_{i}\right\|^{2} \leq\left\|Y^{\prime} \boldsymbol{e}_{i}\right\|^{2} \leq(1+\epsilon / 12)\left\|Y \boldsymbol{e}_{i}\right\|^{2},
\end{aligned}
$$

and $\forall j, 1 \leq j \leq t$,

$$
\begin{aligned}
& \left\|X_{[j,:]}^{\prime}-\tilde{X}_{[j,:]}\right\|_{L_{F}} \leq \delta\left\|X_{[j,:]}^{\prime}\right\|_{L_{F}}, \\
& \left\|Y_{[j,:]}^{\prime}-\tilde{Y}_{[j,:]}\right\|_{L_{F}} \leq \delta\left\|Y_{[j,:]}^{\prime}\right\|_{L_{F}},
\end{aligned}
$$

where $\delta<\frac{\epsilon}{72 n^{2}} \sqrt{\frac{6(1-\epsilon / 12)}{(1+\epsilon / 12)\left(n^{2}-1\right)}}$, we then have

$$
e_{i}^{\top} \boldsymbol{L}_{F}^{-1} \boldsymbol{e}_{i}=\left\|X \boldsymbol{e}_{i}\right\|^{2}+\left\|Y \boldsymbol{e}_{i}\right\|^{2} \stackrel{\epsilon / 3}{\approx}\left\|\tilde{X} \boldsymbol{e}_{i}\right\|^{2}+\left\|\tilde{Y} \boldsymbol{e}_{i}\right\|^{2} .
$$

Proof. According to triangle inequality, one has

$$
\begin{aligned}
& \left|\left\|\tilde{\boldsymbol{X}} \boldsymbol{e}_{i}\right\|-\left\|\boldsymbol{X}^{\prime} \boldsymbol{e}_{i}\right\|\right| \leq\left\|\left(\tilde{\boldsymbol{X}}-\boldsymbol{X}^{\prime}\right) \boldsymbol{e}_{i}\right\| \leq\left\|\tilde{\boldsymbol{X}}-\boldsymbol{X}^{\prime}\right\|_{F} \\
= & \sqrt{\sum_{j=1}^{t}\left\|\tilde{\boldsymbol{X}}_{[j,:]}-\boldsymbol{X}_{[j,:]}^{\prime}\right\|^{2}} \leq \sqrt{\sum_{j=1}^{t} n^{2}\left\|\tilde{\boldsymbol{X}}_{[j,:]}-\boldsymbol{X}_{[j,:]}^{\prime}\right\|_{L_{F}}^{2}} \\
\leq & \sqrt{\sum_{j=1}^{t} \delta^{2} n^{2}\left\|\boldsymbol{X}_{[j,:]}^{\prime}\right\|_{L_{F}}^{2}} \leq \delta n \sqrt{n}\left\|\boldsymbol{X}^{\prime}\right\|_{F} \\
\leq & \delta n \sqrt{n \sum_{i \in F}(1+\epsilon / 12)\left\|\boldsymbol{X} \boldsymbol{e}_{i}\right\|^{2}} \\
\leq & \delta n \sqrt{(1+\epsilon / 12) n \sum_{i \in F} \boldsymbol{e}_{i}^{\top} \boldsymbol{L}_{F}^{-1} \boldsymbol{e}_{i}} \\
\leq & \delta n \sqrt{(1+\epsilon / 12) n\left(n^{2}-1\right) / 6} .
\end{aligned}
$$

Similarly, for $\left\|Y^{\prime} \boldsymbol{e}_{i}\right\|$ and $\left\|\tilde{Y} \boldsymbol{e}_{i}\right\|$, one obtains

$$
\left|\left\|\tilde{Y} \boldsymbol{e}_{i}\right\|-\left\|Y^{\prime} \boldsymbol{e}_{i}\right\|\right| \leq \delta n \sqrt{(1+\epsilon / 12) n\left(n^{2}-1\right) / 6} .
$$

On the other hand,

$$
\begin{aligned}
\left\|X^{\prime} \boldsymbol{e}_{i}\right\|^{2}+\left\|Y^{\prime} \boldsymbol{e}_{i}\right\|^{2} \geq(1-\epsilon / 12)\left(\left\|X \boldsymbol{e}_{i}\right\|^{2}+\left\|Y \boldsymbol{e}_{i}\right\|^{2}\right) \\
\geq(1-\epsilon / 12) \boldsymbol{e}_{i}^{\top} \boldsymbol{L}_{F}^{-1} \boldsymbol{e}_{i} \geq(1-\epsilon / 12) / 2 n
\end{aligned}
$$

For $\left\|X^{\prime} \boldsymbol{e}_{i}\right\|$ and $\left\|Y^{\prime} \boldsymbol{e}_{i}\right\|$, at least one is no less than $\sqrt{\frac{1-\epsilon / 12}{4 n}}$. We only consider the case $\left\|X^{\prime} \boldsymbol{e}_{i}\right\| \geq \sqrt{\frac{1-\epsilon / 12}{4 n}}$, since the $\left\|Y^{\prime} \boldsymbol{e}_{i}\right\| \geq$ $\sqrt{\frac{1-\epsilon / 12}{4 n}}$ can be handled in a similar way.

For $\left\|X^{\prime} \boldsymbol{e}_{i}\right\| \geq \sqrt{\frac{1-\epsilon / 12}{4 n}}$, we distinguish two cases: (i) $\left\|Y^{\prime} \boldsymbol{e}_{i}\right\|>$ $\left\|X^{\prime} \boldsymbol{e}_{i}\right\| \geq \sqrt{\frac{1-\epsilon / 12}{4 n}}$, (ii) $\left\|X^{\prime} \boldsymbol{e}_{i}\right\| \geq\left\|Y^{\prime} \boldsymbol{e}_{i}\right\|$. For case (i), one has

$$
\frac{\left|\left\|\boldsymbol{X}^{\prime} \boldsymbol{e}_{i}\right\|-\left\|\tilde{\boldsymbol{X}} \boldsymbol{e}_{i}\right\|\right|}{\left\|\boldsymbol{X}^{\prime} \boldsymbol{e}_{i}\right\|} \leq 2 \delta n^{2} \sqrt{\frac{(1+\epsilon / 12)\left(n^{2}-1\right)}{6(1-\epsilon / 12)}} \leq \frac{\epsilon}{36},
$$

Based on this result, one further obtains

$$
\begin{aligned}
& \left|\left\|X^{\prime} \boldsymbol{e}_{i}\right\|^{2}-\left\|\tilde{\boldsymbol{X}} \boldsymbol{e}_{i}\right\|^{2}\right| \\
= & \left|\left\|\boldsymbol{X}^{\prime} \boldsymbol{e}_{i}\right\|-\left\|\tilde{\boldsymbol{X}} \boldsymbol{e}_{i}\right\|\right| \cdot\left|\left\|\boldsymbol{X}^{\prime} \boldsymbol{e}_{i}\right\|+\left\|\tilde{\boldsymbol{X}} \boldsymbol{e}_{i}\right\|\right| \\
\leq & \frac{\epsilon}{36}\left(2+\frac{\epsilon}{36}\right)\left\|\boldsymbol{X}^{\prime} \boldsymbol{e}_{i}\right\|^{2} \leq \frac{\epsilon}{12}\left\|X^{\prime} \boldsymbol{e}_{i}\right\|^{2} \leq \frac{\epsilon}{6}\left\|\boldsymbol{X}^{\prime} \boldsymbol{e}_{i}\right\|^{2},
\end{aligned}
$$

which means $\left\|X^{\prime} \boldsymbol{e}_{i}\right\|^{2} \stackrel{\epsilon / 6}{\approx}\left\|\tilde{X} \boldsymbol{e}_{i}\right\|^{2}$. Similarly, we can prove $\left\|Y^{\prime} \boldsymbol{e}_{i}\right\|^{2} \stackrel{\epsilon / 6}{\approx}\left\|\tilde{Y} \boldsymbol{e}_{i}\right\|^{2}$. Combining these relations with the initial condition, one gets $\left\|X \boldsymbol{e}_{i}\right\|^{2}+$ $\left\|Y \boldsymbol{e}_{i}\right\|^{2} \stackrel{\epsilon / 3}{\approx}\left\|\tilde{\boldsymbol{X}} \boldsymbol{e}_{i}\right\|^{2}+\left\|\tilde{\boldsymbol{Y}} \boldsymbol{e}_{i}\right\|^{2}$.

For case (ii), one has

$$
\left\|X^{\prime} \boldsymbol{e}_{i}\right\|^{2} \leq\left\|\boldsymbol{X}^{\prime} \boldsymbol{e}_{i}\right\|^{2}+\left\|Y^{\prime} \boldsymbol{e}_{i}\right\|^{2} \leq 2\left\|\boldsymbol{X}^{\prime} \boldsymbol{e}_{i}\right\|^{2} .
$$

By using a similar process as above leads to

$$
\frac{\left|\left\|Y^{\prime} \boldsymbol{e}_{i}\right\|-\left\|\tilde{Y} \boldsymbol{e}_{i}\right\|\right|}{\left\|X^{\prime} \boldsymbol{e}_{i}\right\|} \leq 2 \delta n^{2} \sqrt{\frac{(1+\epsilon / 12)\left(n^{2}-1\right)}{6(1-\epsilon / 12)}} \leq \frac{\epsilon}{36} .
$$


Based on this obtained result, we further have

$$
\begin{aligned}
& \left|\left\|Y^{\prime} \boldsymbol{e}_{i}\right\|^{2}-\left\|\tilde{Y} \boldsymbol{e}_{i}\right\|^{2}\right| \\
= & \left|\left\|Y^{\prime} \boldsymbol{e}_{i}\right\|-\left\|\tilde{\boldsymbol{Y}} \boldsymbol{e}_{i}\right\|\right| \cdot\left|\left\|Y^{\prime} \boldsymbol{e}_{i}\right\|+\left\|\tilde{\boldsymbol{Y}} \boldsymbol{e}_{i}\right\|\right| \\
\leq & \frac{\epsilon}{36}\left(2+\frac{\epsilon}{36}\right)\left\|X^{\prime} \boldsymbol{e}_{i}\right\|^{2} \leq \frac{\epsilon}{12}\left(\left\|X^{\prime} \boldsymbol{e}_{i}\right\|^{2}+\left\|Y^{\prime} \boldsymbol{e}_{i}\right\|^{2}\right), \\
& \left|\left\|\boldsymbol{X}^{\prime} \boldsymbol{e}_{i}\right\|^{2}-\left\|\tilde{\boldsymbol{X}} \boldsymbol{e}_{i}\right\|^{2}\right| \leq \frac{\epsilon}{12}\left(\left\|\boldsymbol{X}^{\prime} \boldsymbol{e}_{i}\right\|^{2}+\left\|Y^{\prime} \boldsymbol{e}_{i}\right\|^{2}\right) .
\end{aligned}
$$

Combining the above-obtained results, one obtains

$$
\begin{gathered}
\frac{\left|\left(\left\|X^{\prime} \boldsymbol{e}_{i}\right\|^{2}+\left\|Y^{\prime} \boldsymbol{e}_{i}\right\|^{2}\right)-\left(\left\|\tilde{\boldsymbol{X}} \boldsymbol{e}_{i}\right\|^{2}+\left\|\tilde{\boldsymbol{Y}} \boldsymbol{e}_{i}\right\|^{2}\right)\right|}{\left\|X^{\prime} \boldsymbol{e}_{i}\right\|^{2}+\left\|Y^{\prime} \boldsymbol{e}_{i}\right\|^{2}} \\
\leq \frac{\left|\left\|\boldsymbol{X}^{\prime} \boldsymbol{e}_{i}\right\|^{2}-\left\|\tilde{X} \boldsymbol{e}_{i}\right\|^{2}\right|+\left|\left\|Y^{\prime} \boldsymbol{e}_{i}\right\|^{2}-\left\|\tilde{Y} \boldsymbol{e}_{i}\right\|^{2}\right|}{\left\|X^{\prime} \boldsymbol{e}_{i}\right\|^{2}+\left\|Y^{\prime} \boldsymbol{e}_{i}\right\|^{2}} \leq \frac{\epsilon}{6},
\end{gathered}
$$

which, together with the initial condition, leads to (14).

\subsection{Fast algorithm for approximating $\Delta(e)$}

Based on Lemmas 6.3, 6.4 and 6.6, we propose an algorithm OpINIONCoMP approximating $\Delta(e)$ for every candidate edge $e$ in set $Q$, the outline of which is presented in Algorithm 2, and the performance of which is given in Theorem 6.7.

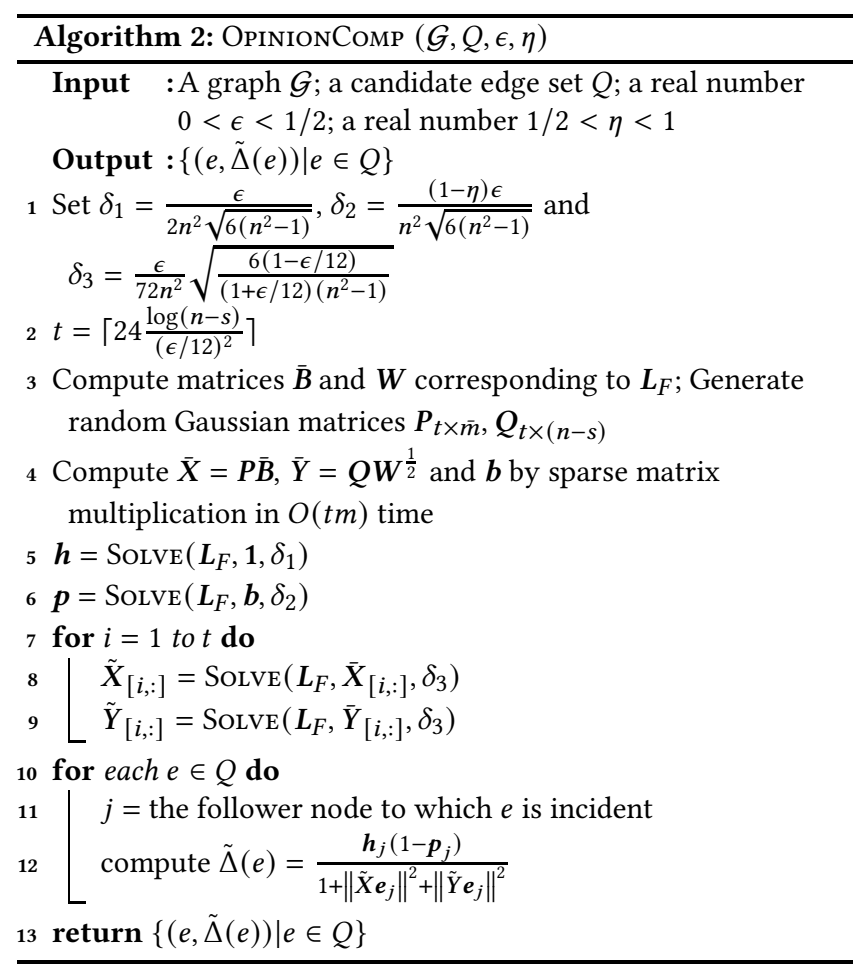

Theorem 6.7. For $0<\epsilon<1 / 2$, the value $\tilde{\Delta}(e)$ returned by OPINIONCOMP satisfies

$$
(1-\epsilon) \Delta(e) \leq \tilde{\Delta}(e) \leq(1+\epsilon) \Delta(e)
$$

with high probability.
Proof. Using Lemmas 6.3, 6.4, and 6.6, one has

as desired.

$$
\frac{|\Delta(e)-\tilde{\Delta}(e)|}{\Delta(e)} \leq \frac{\left|(1+\epsilon / 6)^{2} \Delta(e)-\Delta(e)\right|}{(1-\epsilon / 3) \Delta(e)} \leq \epsilon,
$$

\subsection{Fast Algorithm for Objective Function}

By applying Algorithm 2 to approximate $\Delta(e)$, we propose a fast greedy algorithm $\operatorname{Approx}(\mathcal{G}, Q, k, \epsilon, \eta)$ in Algorithm 3, which solves Problem 1. Algorithm 3 performs $k$ rounds (Lines 2-6). In every round, it takes time $\tilde{O}\left(m \epsilon^{-2}\right)$ to call OpInIONCOMP and update related qualities. Consequently, the time complexity of Algorithm 3 is $\tilde{O}\left(m k \epsilon^{-2}\right)$.

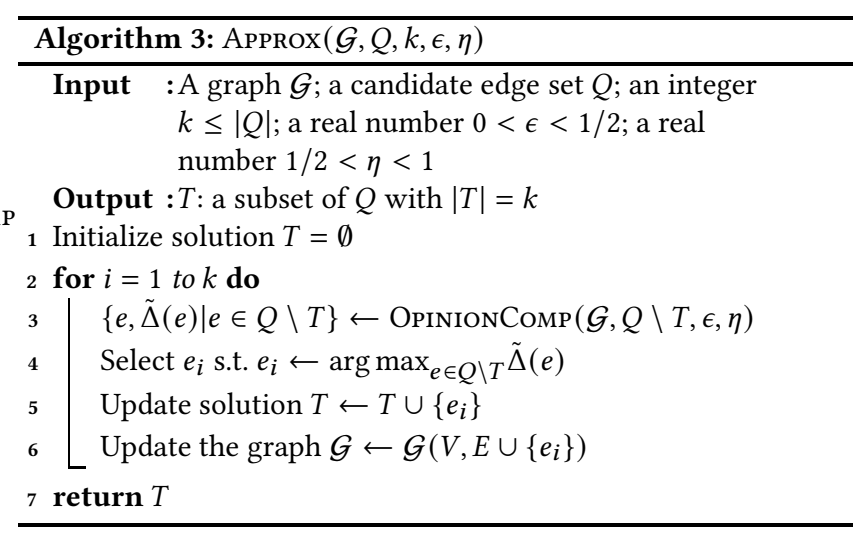
lem 1.

Algorithm 3 yields a $\left(1-\frac{1}{e}-\epsilon\right)$ approximation solution to Prob-

Theorem 6.8. Let $T^{*}$ be the optimal solution to Problem 1, namely,

$$
H\left(T^{*}\right)=\underset{T \subset Q,|T|=k}{\arg \max } H(T) .
$$

Then, the set $T$ returned by Algorithm 3 satisfies

$$
H(T)-H(\emptyset) \geq\left(1-\frac{1}{e}-\epsilon\right)\left(H\left(T^{*}\right)-H(\emptyset)\right) .
$$

Proof. The main difference between algorithms ExACT and Approx is as follows. At each step, ExACT selects an edge with maximum marginal gain, while Approx selects an edge with at least $(1-\epsilon)$ times the maximum marginal gain. Define $T_{i}=\operatorname{Approx}(\mathcal{G}, Q, i, \epsilon, \eta)$, then according to submodularity of $H(\cdot)$,

$$
H\left(T_{i+1}\right)-H\left(T_{i}\right) \geq \frac{1-\epsilon}{k}\left(H\left(T^{*}\right)-H\left(T_{i}\right)\right)
$$

holds for any $i$, which implies

$$
H\left(T_{i+1}\right)-H\left(T^{*}\right) \leq\left(1-\frac{1-\epsilon}{k}\right)\left(H\left(T_{i}\right)-H\left(T^{*}\right)\right) .
$$

Thus, we have

$$
\begin{aligned}
H\left(T^{*}\right)-H\left(T_{k}\right) & \leq\left(1-\frac{1-\epsilon}{k}\right)^{k}\left(H\left(T^{*}\right)-H\left(T_{0}\right)\right) \\
& \leq\left(\frac{1}{e}+\epsilon\right)\left(H\left(T^{*}\right)-H\left(T_{0}\right)\right) .
\end{aligned}
$$

Considering $T_{0}=\emptyset$, we directly finish the proof. 
Table 1: The running time (seconds, $s$ ) and the relative error of Algorithms 1 and 3 on real-world networks for various $\epsilon$.

\begin{tabular}{|c|c|c|c|c|c|c|c|c|c|}
\hline \multirow{2}{*}{ Network } & \multirow{2}{*}{ Nodes } & \multirow{2}{*}{ Edges } & \multicolumn{4}{|c|}{ Running time $(s)$ for ExACT and APPROX } & \multicolumn{3}{|c|}{ Relative error $\left(\times 10^{-2}\right)$} \\
\hline & & & EXACT & $\epsilon=0.3$ & $\epsilon=0.2$ & $\epsilon=0.1$ & $\epsilon=0.3$ & $\epsilon=0.2$ & $\epsilon=0.1$ \\
\hline 494-bus & 494 & 1,080 & 0.837 & 0.655 & 1.382 & 5.314 & 1.07 & 0.75 & 0.51 \\
\hline Bcspwr09 & 1,723 & 4,117 & 10.90 & 3.350 & 6.972 & 27.16 & 4.60 & 2.43 & 1.25 \\
\hline Hamster & 2,426 & 16,630 & 11.27 & 3.377 & 6.936 & 29.27 & 2.21 & 1.48 & 0.83 \\
\hline USGrid & 4,941 & 6,594 & 136.8 & 11.86 & 25.76 & 98.63 & 1.01 & 0.48 & 0.39 \\
\hline Bcspwr10 & 5,300 & 8,271 & 152.5 & 16.23 & 35.12 & 136.1 & 3.74 & 1.75 & 0.91 \\
\hline Reality & 6,809 & 7,680 & 300.9 & 8.715 & 18.40 & 71.56 & 0.50 & 0.43 & 0.17 \\
\hline PagesGovernment & 7,057 & 89,455 & 325.4 & 93.21 & 195.8 & 766.3 & 4.52 & 2.45 & 0.52 \\
\hline HepPh & 11,204 & 117,619 & 1127 & 135.9 & 290.8 & 1120 & 5.30 & 3.89 & 1.95 \\
\hline Anybeat & 12,645 & 49,132 & 1601 & 59.43 & 128.5 & 501.1 & 0.20 & 0.18 & 0.08 \\
\hline Epinions & 26,588 & 100,120 & 12515 & 181.1 & 385.8 & 1568 & 0.70 & 0.35 & 0.09 \\
\hline EmailEU & 32,430 & 54,397 & 22626 & 79.69 & 173.1 & 703.4 & 1.49 & 0.51 & 0.16 \\
\hline GemsecRO & 41,773 & 125,826 & 51236 & 378.3 & 803.2 & 3180 & 1.52 & 1.00 & 0.81 \\
\hline Brightkite & 56,739 & 212,945 & - & 443.3 & 1002 & 3827 & - & - & - \\
\hline LiveMocha & 104,103 & $2,193,083$ & - & 9071 & 20129 & 81203 & - & - & - \\
\hline Douban & 154,908 & 327,162 & - & 1249 & 2732 & 11307 & - & - & - \\
\hline Dblp2010 & 226,413 & 716,460 & - & 2183 & 4837 & 19623 & - & - & - \\
\hline TwitterFollows & 404,719 & 713,319 & - & 2331 & 5261 & 19942 & - & - & - \\
\hline Delicious & 536,108 & $1,365,961$ & - & 5540 & 12433 & 49826 & - & - & - \\
\hline FourSquare & 639,014 & $3,214,986$ & - & 10347 & 23214 & 92170 & - & - & - \\
\hline YoutubeSnap & $1,134,890$ & $2,987,624$ & - & 13689 & 30715 & 124913 & - & - & - \\
\hline
\end{tabular}

\section{EXPERIMENTS}

In this section, we will study the performance of our two heuristic algorithms EXACT and Approx in terms of the effectiveness and the efficiency by implementing experiments on various real-life networks with different scales. The selected data sets of real networks are publicly available in the KONECT [23] and SNAP at website https://snap.stanford.edu, detailed information for whose largest components is presented in the first three columns of Table 1. For the convenience of using the linear solver Solve [24], which can be found at https://github.com/danspielman/Laplacians.jl, all our experiments are programmed in Julia using a single thread, and are run on a machine equipped with $32 \mathrm{G}$ RAM and $4.2 \mathrm{GHz}$ Intel i7-7700 CPU. In our experiments, the candidate edge set $Q$ contains all nonexistent edges satisfying constraints in Section 4.2, for each of which, one end is in set $S_{1}$ and the other end is in set $F$.

\subsection{Effectiveness of Greedy Algorithms}

We first compare the effectiveness of our algorithms with the optimum solution and the solution of a random scheme that randomly choose $k$ edges from $Q$ to add. To this end, we execute experiments on four small real networks: Karate club with 34 nodes and 78 edges, Dolphins with 62 nodes and 159 edges, Tribes with 16 nodes and 58 edges, and FirmHiTech with 33 nodes and 147 edges, which allow us to compute the optimal set of added edges. We randomly select three 0 -leaders and three 1 -leaders. Then, by using different strategies, we add $k=1,2, \ldots, 5$ edges, for each of which, one end is linked to a 1-leader and the other end is connected to a follower. The result is reported in Figure 1, which shows that the solutions returned by our two greedy algorithms are the same as or very close to the optimum solution, and are far better than the random scheme, as well as the theoretical guarantees.

We also compare the results returned by our algorithms with other four baseline schemes: TopCloseness, TopBetweenness, TopPageRank and TopDegree, on relatively large real networks, in order to further demonstrate their effectiveness. The cardinalities of both $S_{0}$ and $S_{1}$ are equal to 10 . For these centrality [34] based
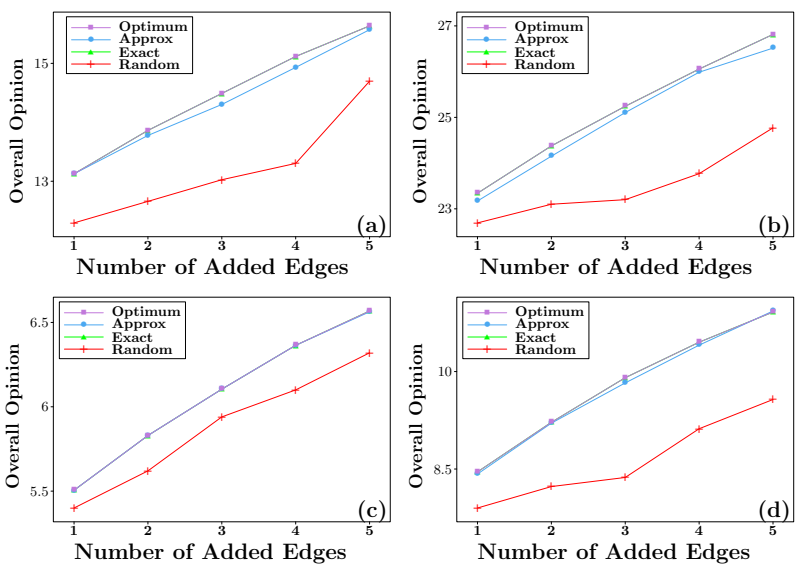

Figure 1: Overall opinion of follower nodes as a function of the number of added edges for our two algorithms, random and the optimum solution on four networks: Karate club (a), Dolphins (b), Tribes (c), and FirmHiTech (d). $\epsilon$ is set to be 0.3 for the approximation algorithm Approx.

baselines, the added edges are just the $k$ edges linking nodes in $S_{1}$ and nodes in the follower set previously nonadjacent to the corresponding 1-leader, which have the highest closeness, betweenness, PageRank, and degree centrality in original network. For each real network, we calculate the overall opinion of followers in the original graph and increase it by generating up to $k=10,20, \ldots, 50$ new edges, applying our greedy algorithms and the four baseline strategies of edge addition. After adding each edge by different methods, we compute and record the overall opinion. The results are plotted in Figure 2, which indicates that for each network, our two greedy algorithms outperform the baseline strategies. 

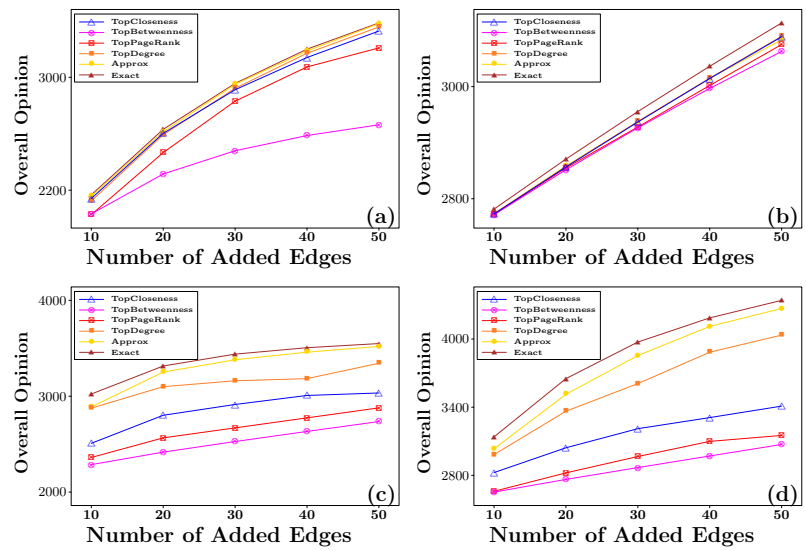

Figure 2: Overall opinion of follower nodes for our two algorithms, and four baseline heuristics on four real netowrks: Reality (a), PagesGovernment (b), USgrid (c), Bcspwr10 (d).

\subsection{Comparison of Performance between our Greedy Algorithms}

As shown above, both of our algorithms ExACT and Approx exhibit good effectiveness, compared with baseline strategies of edge addition. Here we compare the performance of algorithms ExACT and Approx. We first demonstrate that Approx is more efficient than ЕхАст. For this purpose, we compare the running time of algorithms ExACT and Approx on real-life networks in Table 1. For each network, we choose stochastically ten 1-leaders and ten 0 leaders, with the remaining nodes being followers. Then, we add $k=50$ edges between 1-leaders and followers by algorithms ExACT and Approx to maximize the overall opinion. Table 1 shows that for moderate $\epsilon$, Approx is much faster than ExACT, which is more obvious for larger networks. In particular, Approx is scalable to massive networks with one million nodes. For example, for the last eight networks in Table 1, such as YoutubeSnap with over $10^{6}$ nodes, ExAct can't run due to the memory limitation, while Approx still works well.

We proceed to compare the effectiveness of algorithms ExACT and Approx. We define $\gamma$ and $\tilde{\gamma}$ as the increase of overall opinion of followers after adding edges selected, respectively, by ExACT and Approx, and define $\delta=|\gamma-\tilde{\gamma}| / \gamma$ as the relative error between $\gamma$ and $\tilde{\gamma}$. The results of relative errors for different real networks and various parameter $\epsilon$ are presented in Table 1, which demonstrates that for $\epsilon=0.1,0.2$, and 0.3 , relative errors $\delta$ are very small, with the largest value equal to 5.3\%. Thus, the results turns by Approx are very close to those associated with Approx, implying that Approx is both effective and efficient.

\section{CONCLUSIONS}

In this paper, we examined the problem of maximizing the influence of opinion for leaders in a social network with $n$ nodes and $m$ edges by adding $k$ new edges based on the discrete leader-follower DeGroot model for opinion dynamics with $s \ll n$ leaders. The problem is inherently a combinatorial optimization problem that can be applied to various domains. We established the monotonicity and submodularity of the objective function. We put forward two heuristic algorithms. The former returns a $\left(1-\frac{1}{e}\right)$ approximation of the optimal solution in time $O\left(n^{3}\right)$, while the latter has a $\left(1-\frac{1}{e}-\epsilon\right)$ approximation ratio and $\tilde{O}\left(k m \epsilon^{-2}\right)$ complexity. Finally, we performed experiments on real networks of different scales, demonstrating the efficiency and effectiveness of our fast algorithm that is scalable to large-scale networks with more than one million nodes. In future work, we plan to extend or modify our algorithm to other optimization problems for opinion dynamics, such as minimizing risk of conflict, disagreement, and so on.

\section{ACKNOWLEDGEMENTS}

The work was supported by the National Key R \& D Program of China (Nos. 2018YFB1305104 and 2019YFB2101703), the National Natural Science Foundation of China (Nos. 61872093, U20B2051 and U19A2066), the Shanghai Municipal Science and Technology Major Project (Nos. 2018SHZDZX01 and 2021SHZDZX0103), and ZJLab.

\section{REFERENCES}

[1] Rediet Abebe, Jon Kleinberg, David Parkes, and Charalampos E Tsourakakis. 2018. Opinion dynamics with varying susceptibility to persuasion. In Proceedings of the 24th ACM SIGKDD International Conference on Knowledge Discovery and Data Mining. ACM, 1089-1098.

[2] Dimitris Achlioptas. 2001. Database-friendly random projections. In Proceedings of the twentieth ACM SIGMOD-SIGACT-SIGART Symposium on Principles of Database Systems. ACM, 274-281.

[3] Claudio Altafini. 2013. Consensus problems on networks with antagonistic interactions. IEEE Trans. Automat. Control 58, 4 (2013), 935-946.

[4] Victor Amelkin and Ambuj K Singh. 2019. Fighting opinion control in social networks via link recommendation. In Proceedings of the 25th ACM SIGKDD International Conference on Knowledge Discovery and Data Mining. ACM, 677-685.

[5] Brian DO Anderson and Mengbin Ye. 2019. Recent advances in the modelling and analysis of opinion dynamics on influence networks. International fournal of Automation and Computing 16, 2 (2019), 129-149.

[6] Vincenzo Auletta, Diodato Ferraioli, and Gianluigi Greco. 2018. Reasoning about Consensus when Opinions Diffuse through Majority Dynamics.. In Proceedings of the Twenty-Seventh International foint Conference on Artificial Intelligence. 4955.

[7] David Bindel, Jon Kleinberg, and Sigal Oren. 2011. How bad is forming your own opinion?. In Proceedings of the 2011 IEEE 52nd Annual Symposium on Foundations of Computer Science. 57-66.

[8] David Bindel, Jon Kleinberg, and Sigal Oren. 2015. How bad is forming your own opinion? Games and Economic Behavior 92 (2015), 248-265.

[9] T-H. Hubert Chan, Zhibin Liang, and Mauro Sozio. 2019. Revisiting opinion dynamics with varying susceptibility to persuasion via non-convex local search. In Proceedings of the 2019 World Wide Web Conference. ACM, 173-183.

[10] Xi Chen, Jefrey Lijffijt, and Tijl De Bie. 2018. Quantifying and minimizing risk of conflict in social networks. In Proceedings of the 24th ACM SIGKDD International Conference on Knowledge Discovery and Data Mining. ACM, 1197-1205.

[11] Michael B Cohen, Rasmus Kyng, Gary L Miller, Jakub W Pachocki, Richard Peng, Anup B Rao, and Shen Chen Xu. 2014. Solving SDD linear systems in nearly $m \log ^{1 / 2} n$ time. In Proceedings of the Forty-Sixth Annual ACM Symposium on Theory of Computing. ACM, 343-352.

[12] Pierluigi Crescenzi, Gianlorenzo D'angelo, Lorenzo Severini, and Yllka Velaj. 2016. Greedily improving our own closeness centrality in a network. ACM Transactions on Knowledge Discovery from Data 11, 1 (2016), 9.

[13] Gianlorenzo D’Angelo, Martin Olsen, and Lorenzo Severini. 2019. Coverage centrality maximization in undirected networks. In Proceedings of the 33rd AAAI Conference on Artificial Intelligence, Vol. 33. 501-508.

[14] Abhimanyu Das, Sreenivas Gollapudi, and Kamesh Munagala. 2014. Modeling opinion dynamics in social networks. In Proceedings of the 7th ACM International Conference on Web Search and Data Mining. ACM, 403-412.

[15] Morris H. Degroot. 1974. Reaching a consensus. 7. Amer. Statist. Assoc. 69, 345 (1974), 118-121.

[16] Yucheng Dong, Min Zhan, Gang Kou, Zhaogang Ding, and Haiming Liang. 2018. A survey on the fusion process in opinion dynamics. Information Fusion 43 (2018), 57-65. 
[17] Dimitris Fotakis, Dimitris Palyvos-Giannas, and Stratis Skoulakis. 2016. Opinion Dynamics with Local Interactions.. In Proceedings of the Twenty-Fifth International foint Conference on Artificial Intelligence. 279-285.

[18] Noah E Friedkin and Eugene C Johnsen. 1990. Social influence and opinions. Journal of Mathematical Sociology 15, 3-4 (1990), 193-206.

[19] Kiran Garimella, Gianmarco De Francisci Morales, Aristides Gionis, and Michael Mathioudakis. 2017. Reducing controversy by connecting opposing views. In Proceedings of the Tenth ACM International Conference on Web Search and Data Mining. ACM, 81-90.

[20] Aristides Gionis, Evimaria Terzi, and Panayiotis Tsaparas. 2013. Opinion maximization in social networks. In Proceedings of the 2013 SIAM International Conference on Data Mining. SIAM, 387-395.

[21] Peng Jia, Anahita MirTabatabaei, Noah E Friedkin, and Francesco Bullo. 2015 Opinion dynamics and the evolution of social power in influence networks. SIAM Rev. 57, 3 (2015), 367-397.

[22] William B Johnson and Joram Lindenstrauss. 1984. Extensions of Lipschitz mappings into a Hilbert space. Contemp. Math. 26 (1984), 189-206.

[23] Jérôme Kunegis. 2013. Konect: the koblenz network collection. In Proceedings of the 22nd International World Wide Web Conference. ACM, 1343-1350.

[24] Rasmus Kyng and Sushant Sachdeva. 2016. Approximate Gaussian elimination for Laplacians-fast, sparse, and simple. In Proceedings of the IEEE 57th Annual Symposium on Foundations of Computer Science. IEEE, 573-582.

[25] Heidi Ledford. 2020. How Facebook, Twitter and other data troves are revolutionizing social science. Nature 582, 7812 (2020), 328-330.

[26] Huan Li, Stacy Patterson, Yuhao Yi, and Zhongzhi Zhang. 2020. Maximizing the number of spanning trees in a connected graph. IEEE Transactions on Information Theory 66, 2 (2020), 1248-1260.

[27] Huan Li, Richard Peng, Liren Shan, Yuhao Yi, and Zhongzhi Zhang. 2019. Current flow group closeness centrality for complex networks. In Proceedings of the 2019 World Wide Web Conference. ACM, 961-971.

[28] William S Lovejoy and Christoph H Loch. 2003. Minimal and maximal characteristic path lengths in connected sociomatrices. Social Networks 25, 4 (2003), 333-347.

[29] Vassio Luca, Fagnani Fabio, Frasca Paolo, and Ozdaglar Asuman. 2014. Message passing optimization of harmonic influence centrality. IEEE Transactions on Control of Network Systems 1, 1 (2014), 109-120.
[30] Erika Mackin and Stacy Patterson. 2019. Maximizing diversity of opinion in social networks. In Proceedings of 2019 American Control Conference. IEEE, 27282734.

[31] Van Sy Mai and Eyad H Abed. 2019. Optimizing leader influence in networks through selection of direct followers. IEEE Trans. Automat. Control 64, 3 (2019), $1280-1287$.

[32] Judith J McDonald, Michael Neumann, Hans Schneider, and Michael J Tsatsomeros. 1995. Inverse $M$-matrix inequalities and generalized ultrametric matrices. Linear Algebra Appl. 220 (1995), 321 - 341.

[33] Carl D Meyer, Jr. 1973. Generalized inversion of modified matrices. SIAM 7. Appl. Math. 24, 3 (1973), 315-323.

[34] Shogo Murai and Yuichi Yoshida. 2019. Sensitivity analysis of centralities on unweighted networks. In Proceedings of the 2019 World Wide Web Conference. ACM, 1332-1342.

[35] George L Nemhauser, Laurence A Wolsey, and Marshall L Fisher. 1978. An analysis of approximations for maximizing submodular set functions. Mathematical Programming 14, 1 (1978), 265-294.

[36] Hossein Noorazar, Kevin R Vixie, Arghavan Talebanpour, and Yunfeng Hu. 2020. From classical to modern opinion dynamics. International fournal of Modern Physics C 31, 07 (2020), 2050101.

[37] Liren Shan, Yuhao Yi, and Zhongzhi Zhang. 2018. Improving information centrality of a node in complex networks by adding edges. 27th International foint Conference on Artificial Intelligence, 3535-3541.

[38] Daniel A Spielman and Shang-Hua Teng. 2014. Nearly linear time algorithms for preconditioning and solving symmetric, diagonally dominant linear systems. SIAM 7. Matrix Anal. Appl. 35, 3 (2014), 835-885.

[39] Pinghua Xu, Wenbin Hu, Jia Wu, and Weiwei Liu. 2020. Opinion maximization in social trust networks. In Proceedings of the 29th International foint Conference on Artificial Intelligence. 1251-1257.

[40] Yuhao Yi, Timothy Castiglia, and Stacy Patterson. 2019. Shifting opinions in a social network through leader selection. arXiv:1910.13009

[41] Yuhao Yi and Stacy Patterson. 2020. Disagreement and polarization in two-party social networks. IFAC-PapersOnLine 53, 2 (2020), 2568-2575.

[42] Ercan Yildiz, Asuman Ozdaglar, Daron Acemoglu, Amin Saberi, and Anna Scaglione. 2013. Binary opinion dynamics with stubborn agents. ACM Transactions on Economics and Computation 1, 4 (2013), 1-30. 